\title{
ON EXPOSED AND FARTHEST POINTS IN NORMED LINEAR SPACES
}

\author{
M. EDELSTEIN and J. E. LEWIS
}

(Received 19 May 1969)

Communicated by B. Mond

Let $S$ be a nonempty subset of a normed linear space $E$. A point $s_{0}$ of $S$ is called a farthest point if for some $x \in E$,

$$
\left\|x-s_{0}\right\|=\sup \{\|x-s\|: s \in S\} .
$$

The set of all farthest points of $S$ will be denoted far $(S)$. If $S$ is compact, the continuity of distance from a point $x$ of $E$ implies that far $(S)$ is nonempty.

A point $s_{1}$ of $S$ is said to be an exposed point if there is some continuous linear functional $g$ with

$$
g\left(s_{1}\right)>g(s), \quad \text { for each } s \in S \sim\left\{s_{1}\right\} .
$$

The set of all exposed points is denoted $\exp (S)$; and if $S$ is compact, exp (S) is nonempty (c.f. Klee [4]).

In general, $\exp (S)$ need not be identical with far $(S)$, even for compact $S$. In this connection, Bernau [2] endeavored to show that every normed linear space of dimension greater than one contains a compact convex $S$ for which $\exp (S)$ fails to be a subset of far $(S)$.

The first part of this note is concerned with the relationship between $\exp (S)$ and $\operatorname{far}(S)$ for compact convex subsets. In the second part, we briefly discuss the same problem when the compactness condition is relaxed.

The notations $B(x, r)$ and $\bar{B}(x, r)$ are used to denote, respectively, the subsets $\{y:\|y-x\|<r\}$ and $\{y:\|y-x\| \leqq r\}$. All spaces are assumed to be real spaces.

\section{Exposed points and farthest points of compact convex subsets}

If $S$ is a nonempty compact convex subset of a strictly convex normed linear space, then clearly $\exp (S) \supset$ far $(S)$. In this section we will be interested in the possibility of obtaining the reverse inclusion (for spaces which are not necessarily strictly convex).

For an arbitrary nonempty set, $I$, the linear space $m_{0}(I)$ consists of all those bounded real-valued functions $x$ for which $\{i \in I:|x(i)|>0\}$ is at most countable. It is readily seen, and well known, that with the usual sup norm, $m_{0}(I)$ is a Banach space. 
1. Proposition. Let I be an uncountable set, and let $K$ be a compact subset of $m_{0}(I)$. Then all points of $K$ are equidistant from some point $z$ of $m_{0}(I)$.

Proof. Let $P: m_{0}(I) \rightarrow 2^{I}$ be the (set valued) mapping defined by

$$
P(x)=\{i \in I:|x(i)|>0\} .
$$

Note that if $K \subset m_{0}(I)$ is bounded and if there is some point $i_{0} \in I \sim P[K]$, then $K$ is equidistant from the point $z$, where $z$ is defined by

$$
\begin{aligned}
z(i) & =0, \quad i \neq i_{0} \\
z\left(i_{0}\right) & =\sup \{\|x\|: x \in K\} .
\end{aligned}
$$

Consequently, since $I$ is uncountable, it suffices to show that for compact $K, P[K]$ is countable. Clearly, $P[K]$ would be countable if for any positive integer $n$ there is a countable subset $M_{n} \subset I$ such that

$$
x \in K \text { and }|x(i)| \geqq \frac{1}{n} \Rightarrow i \in M_{n} .
$$

For each positive integer $n$, the compactness of $K$ guarantees the existence of a finite subset $A_{n} \subset K$ such that $K \subset A_{n}+B(\overline{0}, 1 / n)$. Now take $M_{n}$ to be the subset $P\left[A_{n}\right]$, which is clearly countable. To show that (1) holds, take $i \in P[K] \sim M_{n}$. Since $K \subset A_{n}+B(\overline{0}, 1 / n)$, and since $y(i)=0$ for any $y \in A_{n}$, it follows that for each $x$ in $K$ we must have $|x(i)|<1 / n$. That is,

$$
i \notin M_{n} \Rightarrow|x(i)|<\frac{1}{n} \quad \text { for each } x \in K,
$$

which completes the proof of the proposition.

With uncountable $I$, a consequence of the preceeding proposition is that every compact subset $K$ of $m_{0}(I)$ has the property that each point of $K$ is a farthest point, and so $\exp (K) \subset \operatorname{far}(K)$. Thus, the theorem of [2], claiming that every normed linear space of dimension greater than one contains a compact convex set with an exposed point which is not a farthest point, is not valid. The reason for the failure of that theorem is that in the absence of some sort of uniformity of the norm, one cannot restrict attention to two dimensional subspaces. In the presence of such conditions, we have the following two propositions.

2. Proposition. Let $E$ be a uniformly convex normed linear space of dimension $\geqq 2$. Then $E$ contains a two dimensional compact convex set $K$ such that $\exp (K)$ $\sim \operatorname{far}(K) \neq \phi$.

Proof. We show, in fact, that if $F$ is any two dimensional subspace of $E$, then $F$ contains a compact convex. $K$ with $\exp (K) \sim$ far $(K) \neq \phi$. Let $x$ and $y$ in $F$ be chosen so that $\|x\|=\|y\|=1$ and

$$
\inf \{\|y-\lambda x\|: \lambda \in \mathbb{R}\}=1 \text {. }
$$


For each positive integer $n$ define the points $z_{n}$ and $z_{n}^{\prime}$ of $F$ by

$$
z_{n}=\delta\left(\frac{1}{n^{2}}\right) y+\frac{1}{n} x \quad z_{n}^{\prime}=\delta\left(\frac{1}{n^{2}}\right) y-\frac{1}{n} x,
$$

where $\delta$ is the modulus of convexity of the space (i.e., $\delta$ is the function defined for all $0<\varepsilon \leqq 2$ by

$$
\left.\delta(\varepsilon)=\inf \left\{1-\frac{1}{2}\|x+y\|:\|x-y\| \geqq \varepsilon, x, y \in \bar{B}(\overline{0}, 1)\right\}\right) .
$$

Let $K$ be the closed convex hull of $\left\{z_{1}, z_{2}, \cdots\right\} \cup\left\{z_{1}^{\prime}, z_{2}^{\prime}, \cdots\right\}$. It is clear that $K$ is two dimensional, and compact. The construction of the points $z_{n}$ and $z_{n}^{\prime}$ shows that $\overline{0} \in \exp (K)$.

Suppose that the closed ball $\bar{B}(w,\|w\|)$ contains the set $K$ (i.e., suppose $\overline{0} \in \operatorname{far}(K))$. Since, if $\overline{0}$ is farthest from $w$, it is also farthest from $t w$ for $t>1$, we may assume that $\|w\|>1$. Choose any $n>\|w\|$. Since we have assumed that $K \subset \bar{B}(w,\|w\|)$, then $\left[\|w\|^{-1}\left(z_{n}-w\right),\|w\|^{-1}\left(z_{n}^{\prime}-w\right)\right] \subset \bar{B}(\overline{0}, 1)$. This implies

$\delta\left(\left\|\frac{z_{n}-w}{\|w\|}-\frac{z_{n}^{\prime}-w}{\|w\|}\right\|\right) \leqq 1-\frac{1}{2}\left\|\frac{z_{n}-w}{\|w\|}+\frac{z_{n}^{\prime}-w}{\|w\|}\right\|$

$$
=1-\frac{1}{2}\left\|\frac{2 y}{\|w\|} \delta\left(\frac{1}{n^{2}}\right)-\frac{2 w}{\|w\|}\right\| \leqq \frac{1}{\|w\|} \delta\left(\frac{1}{n^{2}}\right) \leqq \frac{1}{n^{2}}
$$

and so

$$
\frac{1}{\|w\|}\left\|z_{n}-z_{n}^{\prime}\right\|=\left\|\frac{z_{n}-w}{\|w\|}-\frac{z_{n}^{\prime}-w}{\|w\|}\right\| \leqq \frac{1}{n^{2}} .
$$

This contradicts the fact that $1 /\|w\|\left\|z_{n}-z_{n}^{\prime}\right\|=1 /\|w\| \cdot 2 / n>1 / n^{2}$, which proves that $\overline{0} \in \exp (K) \sim \operatorname{far}(K)$. In the proof of the above proposition we obtained a compact convex set $K$ having at least one exposed point which is not a farthest point. It is now natural to ask whether one can construct such sets $K$ with far $(K)$ $\cap \exp (K)=\phi$. In strictly convex spaces this is, of course, impossible. The next proposition describes a class of spaces in which sets $K$ as above do indeed exist.

3. Proposition. Let E be a strictly convex normed linear space, and let $G$ be the space $E \times \mathbb{R}$ with the norm $\|(x, r)\|=\max (\|x\|,|r|)$. Then $G$ contains a two dimensional compact convex subset $K$ for which $\exp (K) \cap \operatorname{far}(K)=\phi$.

Proof. We will identify $E$ and $\mathbb{R}$ in the usual way with the subspaces $\{(x, 0)$ : $x \in E\}$ and $\{(\overline{0}, r): r \in \mathbb{R}\}$ of $G$. Let $F$ be a two dimensional subspace containing $\mathbb{R}$. If $z$ is any point of $G$ and if $a>0$, it can be verified that $\bar{B}(z, a) \cap F$ is either empty, or a straight line segment parallel to $\mathbb{R}$, or a rectangle whose edges are parallel to $\mathbb{R}$ and to $F \cap E$. Now let $B_{1}=\bar{B}(\overline{0}, 1) \cap F$, and $B_{2}=\{(x, r) \in F$ : $\left.\|\left. x\right|^{2}+|r|^{2} \leqq 1\right\}$, and let $K$ be the compact convex set $B_{1}+B_{2}$. It is easy to see that far $(K)$ consists of the straight line segments in the relative boundary of $K$ in $F$, and, since $K$ is smooth in $F$, that none of these straight line segments contains an exposed point. That is, $\exp (K) \cap \operatorname{far}(K)=\phi$. 
Without demanding that the compact convex set $K$ be of two dimensions, there is another class of spaces in which $\exp (K)$ may fail to be a subset of far $(K)$.

4. Proposition. Let E be a separable normed linear space of dimension $\geqq 2$. Then $E$ contains a compact convex subset $K$ with $\exp (K) \sim \operatorname{far}(K) \neq \phi$.

Proof. First, suppose that $E$ is reflexive. Then there is some point $y$ in $\bar{B}(\overline{0}, 1)$ which is exposed by a continuous linear functional $g$ (c.f. [5]). Due to the choice of $g$ and $y$ note that $g^{-1}[0] \cap \bar{B}(y, 1)=\{\overline{0}\}$.

Let $S=\left\{x \in g^{-1}[0]:\|x\|=1\right\}$, and let $\left\{x_{n}: n=1,2, \cdots\right\}$ be a dense subset of $S$. Then for each pair of integers $(m, n)$ with $m \geqq n \geqq 1$ the straight line segment $\left[m^{-2} x_{n}, m^{-2} x_{n}+y\right]$ intersects the closed convex set $\bar{B}(y, 1)$, since $\left(m^{-2} x_{n}+y\right) \in \bar{B}(y, 1)$. Since $m^{-2} x_{n} \notin \bar{B}(y, 1)$, there is a unique smallest positive number $t_{m, n}$ such that $\left(m^{-2} x_{n}+t_{m, n} y\right) \in \bar{B}(y, 1)$. Using the numbers $t_{m, n}$, for each pair of integers $(m, n)$ with $m \geqq n \geqq 1$ we define $z_{m, n}$ to be the point $m^{-1}\left(x_{n}+t_{m, n} y\right)$ and $z_{m, n}^{\prime}$ to be the point $m^{-1}\left(-x_{n}+t_{m, n} y\right)$.

The vectors $z_{m, n}$ and $z_{m, n}^{\prime}$ can be arranged to form a sequence converging to $\overline{0}$. Let $K$ be the closed convex hull of this sequence. It follows that $K$ is compact, and that $g^{-1}[0] \cap K=\{\overline{0}\}$, i.e. $\overline{0} \in \exp (K)$.

If $E$ is non-reflexive, let $g$ be a continuous linear functional which fails to attain its norm on the closed unit ball of $E$, and choose any point $y$ in $E \sim g^{-1}[0]$. Then define $S$ and $\left\{x_{n}: n=1,2, \cdots\right\} \subset S$ as in the reflexive case. For each pair of integers $(m, n)$ with $m \geqq n \geqq 1$, define $t_{m, n}$ to be $m^{-1}$. Then construct the points $z_{m, n}$ and $z_{m, n}^{\prime}$ and the convex set $K$ in exactly the same way as before. Again, $K$ is compact and $g^{-1}[0] \cap K=\{\overline{0}\}$.

In both the reflexive and non-reflexive cases, the point $\overline{0}$ is a smooth point of $K$. To see this, let $f$ be any functional which is linearly independent of $g$, and choose some $x_{s} \in\left\{x_{n}: n=1,2, \cdots\right\}$ for which $f\left(x_{s}\right) \neq 0$. Suppose that $f$ supports $K$ at $\overline{0}$. We may assume that $f[K] \subset(-\infty, 0]$. Then, for all $m \geqq s$, we would have

$$
f\left(z_{m, s}\right) \leqq 0 \text { and } f\left(z_{m, s}^{\prime}\right) \leqq 0 .
$$

Thus $f\left(x_{s}+t_{m, s} y\right) \leqq 0$ and $f\left(-x_{s}+t_{m, s} y\right) \leqq 0$, and since $t_{m, s} \rightarrow 0$ as $m \rightarrow \infty$ it follows that $f\left(x_{s}\right)=0$, establishing the contradiction.

If $\bar{B}(w,\|w\|)$ contains $K$ (i.e. if $\overline{0}$ is a point of $K$ farthest from $w$ ) the smoothness of $K$ at $\overline{0}$ implies that $g$ supports $\bar{B}(w,\|w\|)$ at $\overline{0}$. Consequently, $g$ would support the closed unit ball at $w /\|w\|$.

In the non-reflexive case this contradicts the definition of $g$, and so, for the non-reflexive case, $\overline{0} \notin$ far $(K)$. In the reflexive case, the choice of $g$ and $y$ would imply that the point $w$ is in the one dimensional subspace $L$ spanned by $y$, and so to show that $\overline{0} \notin \operatorname{far}(K)$ it will be sufficient to show that no point of $L$ admits $\overline{0}$ as a farthest point in $K$. Clearly, by the construction of $K$, we need only prove this for points $w=m y$ where $m=2,3, \cdots$. For such a point $w$ we have:

$$
m^{-1}\left\|z_{m, n}-w\right\|=\left\|m^{-1} z_{m, n}-y\right\|=\left\|m^{-2} x_{n}+t_{m, n} m^{-1} y-y\right\| .
$$


Since $t_{m, n} m^{-1}<t_{m, n}$, the definition of the numbers $t_{m, n}$ implies, then, that $m^{-1}|| z_{m, n}-w \|>1$. Thus, $\left\|z_{n, m}-w\right\|>m=\|w\|$, which shows that $\overline{0}$ is not a point of $K$ farthest from $w$, and completes the proof of the proposition.

REMARK. In general, the compact convex set $K$ in the above proof cannot be finite dimensional. For if $\tilde{c}_{0}$ is the subspace of the separable space $c_{0}$ which consists of all finite sequences, then each compact convex finite dimensional subset of $\tilde{c}_{0}$ is equidistant from some point.

In connection with the problem of finding spaces for which $\exp (K) \subset \operatorname{far}(K)$ for all compact convex subsets $K$, we mention that, in a certain sense, this inclusion 'almost' holds for any smooth normed linear space: In [3] the notion of a $\sigma$ boundedly exposed point was introduced. This is a point $s$ of a subset $S$ such that there is a sequence of open balls $B_{n}$ with the following properties:

(i) $B_{n} \subset B_{n+1}(n=1,2, \cdots)$

(ii) the diameter of $S \sim B_{n}$ tends to zero as $n$ increases

(iii) $s \in b d r y\left(\bigcup_{1}^{\infty} B_{n}\right)$.

The set of all $\sigma$-boundedly exposed points is denoted $\sigma b(S)$. A $\sigma$-boundedly exposed point may be thought of as an exposed point which is 'asymptotically' a farthest point, and it is in this sense that the inclusion $\exp (K) \subset$ far $(K)$ almost holds. That is:

5. Proposition. If $K$ is a compact convex subset of a smooth normed linear space, then $\exp (K)=\sigma b(K)$. Consequently $\exp (K) \subset f a r(K)$.

Proof. Note first that each exposed point of $K$ is strongly exposed, i.e. if $x \in \exp (K)$, then there is some continuous linear functional $f$ such that $\left\{x_{n}\right\} \subset K$ and $f\left(x_{n}\right) \rightarrow f(x)$ implies $x_{n} \rightarrow x$. The remainder of the proof is similar to the second part of the proof of theorem 2.1 of Klee [4] and therefore omitted.

\section{Exposed points and farthest point of closed and bounded convex sets}

Although proposition 1 shows that $m_{0}(I)$, for uncountable $I$, is a space in which $\exp (K) \subset \operatorname{far}(K)$ for all compact convex $K$, we note that $m_{0}(I)$ is not strictly convex (nor even isomorphic to a strictly convex space), and so there are subsets $K$ for which far $(K)$ fails to be contained in $\exp (K)$. In particular, the results of section 1 do not answer the following questions:

Is there a normed linear space in which the notion of exposed point and farthest point coincide for

(1) all finite dimensional compact convex subsets?

(2) all compact convex subsets?

(3) all weakly compact convex subsets? 
Question (2) is really the central question raised by Bernau [2]. The following proposition shows that the two notions never coincide for arbitrary closed and bounded subsets of a normed linear space, and so shows that in a reflexive space, question (3) has a negative answer.

6. Proposition. Let E be a normed linear space. Then E contains a closed and bounded convex subset $C$ for which $\exp (C) \sim \operatorname{far}(C) \neq \phi$.

Proor. If $E$ is reflexive (non-reflexive) let $g$ be a continuous linear functional which supports the closed unit ball of $E$ at an exposed point $y$, [5] (let $g$ be a continuous linear functional which fails to support the closed unit ball, and choose $\left.y \notin g^{-1}[0]\right)$.

Let $D$ be the closed unit ball of $g^{-1}[0]$, and define the subsets $A_{n} \subset E \sim$ $g^{-1}[0]$ by $A_{n}=n^{-1}\left(D+t_{n} y\right)$, where in the reflexive case $t_{n}$ is chosen so that $t_{n}>0$ and $n^{-2} D+t_{n} y$ is not contained in $\bar{B}(y, 1)$; and in the non-reflexive case, $t_{n}=n^{-1}$.

Setting $C=\overline{c o}\left(\bigcup A_{n}\right)$ we see that $C$ is closed and bounded, and, in a manner similar to the proof of proposition $4, \overline{0} \in \exp (C) \sim \operatorname{far}(C)$.

The following, though of independent interest, also serves to show that the above proposition may be fulfilled because far $(C)$ is void, even in an inner product space.

7. Proposition. There exists a closed and bounded convex set $C$ in $l_{2}$ with the property that every point in a dense subspace, $\tilde{l}_{2}$, has no farthest point in $C$.

Proof. Let $\left\{e_{n}: n=1,2, \cdots\right\}$ be the usual orthonormal basis for $l_{2}$. The subspace $\tilde{l}_{2}$ will consist of all members $y \in l_{2}$, for which $\left\{n:\left\langle y, e_{n}\right\rangle \neq 0\right\}$ is finite.

Let $A_{n}$ be the set of all ordered $n$-tuples $\left(k_{1}, k_{2}, \cdots k_{n}\right)$ of positive integers with $k_{1}<k_{2}<\cdots<k_{n}$, and let $A_{w}$ be the set of all sequences $\left(k_{1}, k_{2}, \cdots\right)$ of positive integers with $k_{1}<k_{2}<\cdots$, and define $A$ to be $A_{w} \cup\left(\bigcup_{n=1}^{\infty} A_{n}\right)$. Define the mapping $\varphi: A \rightarrow l_{2}$ by

if $n=k_{i}$, the $i^{\text {th }}$ element of $a$,

$$
\left\langle\phi(a), e_{n}\right\rangle=\frac{1}{2^{i-1}}\left(1-\frac{1}{k_{i}}\right)
$$

$$
\left\langle\varphi(a), e_{n}\right\rangle=0 \quad \text { otherwise. }
$$

Clearly, $\varphi$ is well defined and the set $S=\varphi[A]$ is bounded. We claim that $S$ is weakly compact. Since $S$ is bounded and $l_{2}$ is reflexive it suffices to show that $S$ is weakly closed. Let $y \in l_{2} \sim S$. Then as can be readily seen there must be some smallest positive integer $m$ for which $\sum_{i=1}^{m}\left\langle e_{i}, y\right\rangle e_{i} \notin S$. For each $i=1,2, \cdots, m$, let $S_{i}$ consist of those members of $S$ whose $i^{\text {th }}$ coordinate differs from $\left\langle y, e_{i}\right\rangle$. It is easy to check that $S=\bigcup_{i=1}^{m} S_{i}$. Now define the numbers

$$
\delta_{i}=\inf \left\{\left|y_{i}-\left\langle s, e_{i}\right\rangle\right|: s \in S_{i}\right\} \text {. }
$$


Note that for a given integer $i$, there are at most $i+1$ members of the set $\left\{\left\langle s, e_{i}\right\rangle\right.$ : $s \in S\}$, for the integer $i$ can occupy only one of the first $i$ places in each member $a$ of $A$. By the definition of the sets $S_{i}$, we then see that $\delta_{i}$ is the minimum of a finite subset of positive numbers, and so $\left.\delta_{i}\right\rangle 0$. Since $\left\langle\cdot, e_{i}\right\rangle$ is a continuous linear functional on $l_{2}$, the subset $W_{i}$ defined by $\left\{x \in l_{2}:\left|\left\langle x, e_{i}\right\rangle-y_{i}\right|<\delta_{i}\right\}$ is a weakly open neighborhood of $y$. Then $W=\bigcap_{i=1}^{m} W_{i}$ is a weakly open neighborhood of $y$ which is disjoint from $S$. This completes the proof that $S$ is weakly compact.

Now let $C=\overline{c o}(S)$ and suppose that $x \in \tilde{l}_{2}$ admits a farthest point in $C$, say $y$. Since $l_{2}$ is strictly convex, it follows that $y$ is an extreme point of $C$, and since $S$ and $\overline{c o}(S)$ are weakly compact subsets of $l_{2}$, it follows that $y$ is a point of $S$. Since there must be an integer $N$ such that $\left\langle x, e_{n}\right\rangle=0$ for all $\left.n\right\rangle N$, and since $y=\varphi(a)$ for some $a \in A$, it follows that $y$ is not a point of $S$ farthest from $x$ : If $a=\left(k_{1}, k_{2}, \cdots, k_{m}\right)$, take $\bar{a}$ to be the $(m+1)$-tuple $\left(k_{1}, k_{2}, \cdots, k_{m}, k_{m+1}\right)$ where $k_{m+1}=\max \left(k_{m}+1, N\right)$. If $a$ is a sequence, i.e., $a=\left(k_{1}, k_{2}, \cdots\right)$ find the largest integer $k_{m}$ such that $k_{m}<N$, and define $\vec{a}$ to be the sequence $\left(k_{1}, k_{2}, \cdots\right.$, $\left.k_{m}, k_{m}+1, k_{m+1}+1, k_{m+2}+1, \cdots\right)$. Since

$$
\|x-a\|^{2}=\sum\left|x_{n}-\frac{1}{2^{i-1}}\left(1-\frac{1}{n}\right)^{2}\right|,
$$

where $k_{1}=n$, it is easy to see that, $\|x-\bar{a}\|>\|x-a\|$, which proves that $x$ does not admit a farthest point in $C$.

Remark. In the (incomplete) inner product space $\tilde{l}_{2}$ the closed convex set $C \cap \tilde{l}_{2}$ fails to have farthest points. Indeed, assuming $x \in \tilde{l}_{2}$ and $y \in C \cap \tilde{l}_{2}$ with $y$ farthest frem $x$, then there must be $z \in C$ with $\|z-x\|>y-x \|$, and this inequality would hold for all points in a small enough neighborhood of $z$. Since $C \cap l_{2}$ is dense in $C$, it follows that there must be a $\bar{z} \in C \cap \tilde{l}_{2}$ with $\|\bar{z}-x\|>$ $\|y-x\|$.

Note that this must fail for any complete inner product space, for any closed and bounded subset of a reflexive, locally uniformly convex space $E$ must have farthest points; in fact, there is a set of the second category in $E$ each point of which admits a farthest point in a given closed and bounded subset (sec Asplund [1]). The preceeding proposition shows that in general we cannot expect more than a subset of the second category.

We also mention that $C \cap \tilde{l}_{2}$ has exposed points, for if $a \in \bigcup_{n=1}^{\infty} A_{n}$, with $a=\left(k_{1}, k_{2}, \cdots, k_{n}\right)$ then the functional $\left\langle, e_{k_{1}}\right\rangle$ strongly exposes $\varphi(a)$ in $S$, hence in $C \cap \tilde{l}_{2}$.

The question arises as to whether or not there is some Banach space $E$ which contains a closed and bounded convex subset $C$ with far $(C)=\phi$. If $c_{0}$ is renormed to be strictly convex, and $C$ is the original closed unit ball of $c_{0}$, then, in the new norm $C$ fails to have farthest points. However, the set $C$ fails also to have extreme 
points, and so it is still open as to whether or not there is a Banach space $E$ with a closed and bounded convex subset $C$ for which $\exp (C) \sim \operatorname{far}(C) \neq \phi$ by virtue of the fact that far $(C)=\phi$.

This research was supported by the National Research Council of Canada, Grant A-3999.

\section{References}

[1] E. Asplund, 'Farthest points in reflexive locally uniformly rotund Banach spaces', Israel J. of Math. 4 (1966), 213-216.

[2] S. J. Bernau, 'On bare points', J. Aust. Math. Soc. 9 (1969), 25-28.

[3] M. Edelstein, 'On some special types of exposed points of closed and bounded sets in Banach spaces', Indag. Math. 28 (1966), 360-363.

[4] V. Klee, 'Extremal structure of convex sets II', Math. Zeitschr. 69 (1958), 90-104.

[5] J. Lindenstrauss, 'On non-separable reflexive Banach spaces', Bull. Amer. Math. Soc. 72 (1967), 967-970.

Dalhousie University

Halifax, Nova Scotia 